\title{
Nefrologia clinica: dalla stretta di mano all'EBM attraverso la bioetica
}

\author{
Mario Timio
}

Dipartimento di Medicina Interna e Dialisi, Ospedale di Foligno, Perugia e Direzione Scientifica, Centro di Bioetica della Regione Umbria, Perugia

\begin{abstract}
HANDSHAKE AS THE FIRST STEP IN ETHICAL MEDICINE
Abstract. The concept of medical progress is usually taken for granted; however, in some sense, it gets out of hand if the value of the solid relationship between doctor and patient is underestimated. Such an interaction starts with a strong handshake and a pat on the shoulder: two sings for a correct start of a bioethical approach. Patients usually evaluate the doctor by non-professional norms, which may be influenced by the patient's cultural background and the lay referral process. Thus, to some extent, the doctor must perform in accordance with the patient's expectations, which may require him to adapt to new bioethical norms. Whether the patient will return or follow the physician's advice depends on the patient's evaluation of how he performed his role, mainly in ethical terms. Here we report three cases of nephrological interest relating to the expectations communicated to the doctors, together with their response when dealing with these expectations.
\end{abstract}

Key words: Hand-shake, Ethical approach, Narrative medicine

Conflict of interest: None.

Financial support: None.

Accettato: 14 Settembre 2014

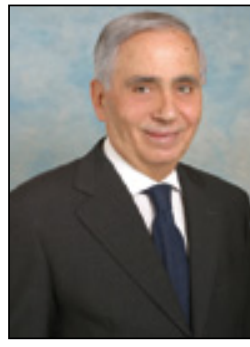

Mario Timio

\section{Presentazione}

È stato il mio Maestro, il Professor Giovanni Gigli a trasmettermi, tra i numerosi insegnamenti professionali, il valore della stretta di mano alla persona malata. "Piccola cosa per il medico" - egli asseriva - "ma grande per il malato". Egli argomentava inoltre che la stretta di mano dovrebbe essere contemporanea alla presentazione: conoscere il nome e cognome dell'ammalato contribuisce a creare confidenza e simpatia, riducendo la tensione dell'incontro, specialmente se sono trasmesse notizie non buone, come la necessità di iniziare la dialisi. Nella pratica clinica ho seguito sempre l'insegnamento del Professor Gigli; da subito ho realizzato l'importanza del piccolo grande gesto che va nel cuore del rapporto medico-malato. La profondità del gesto è stata magnificata dalla lettura di un libro di Robert Buckman: How to break bad news (1). Un capitolo dedicato alla "stretta di mano in medicina" mi ha particolarmente colpito, facendo riemergere nella sua pienezza il profetico messaggio del Maestro mio e di tanti altri medici. Il medico con la stretta di mano riscopre e realizza la sua umanità, vicinanza, solidarietà, sorrette da pilastri di professionalità e fondamenta della bioetica laica. Nel periodo di grande avanzamento tecnologico quale si verifica anche in nefrologia e dialisi - la stretta di mano è destinata a rompere la barriera tra medico e malato e il suo effetto favorevole è potenziabile, come ricorda ancora Buckman, da un cordiale "Come va Signor Bianchi?" È uno spreco di tempo in una fase storica in cui tutto sembra essere fast, dal pasto alla visita medica, passando attraverso le varie tappe veloci della quotidianità? Credo di no, poiché in questo modo il medico si impegna il più possibile a sdrammatizzare il suo incontro con l'ammalato, ottenendo il primo successo della propria cura (2). Qualche lettore, specialmente se giovane può dire: "Ma dove vivi, non sai che essenzialmente negli ambulatori i tempi di visita sono contingentati e quindi bisogna essere celeri per permettere a tutti gli astanti 'prenotati ${ }^{\text {' }} \mathrm{di}$ essere visitati?". Certo che lo so, ma l'insegnamento di Gigli sulla stretta di mano induce a pensare - proprio malgrado i tempi - che il medico nell'esercizio professionale porta con sé la sua vita, non la lascia a casa. E non lascia a casa la sua vocazione, la sua missione di essere sempre dalla parte del malato, a cominciare dalla stretta di mano. E dalla stretta di mano si passa da una parte alla conoscenza scientifica basata sull'Evidence Based Medicine (EBM) e dall'altra si penetra nell'intimo dei segreti e della storia dell'uomo malato. Di 
fronte al medico tutti si spogliano per l'immensa fiducia che in lui ripongono, attivata da una forte stretta di mano. A mio avviso è sempre valida l'immagine del dottore tratta da un libro di bioetica: "Un medico aperto a tutti, che si sacrifica per gli altri e per la salute pubblica, che entra nella casa e nella vita di tutti, un medico curante ogni uomo e in ogni situazione, un medico sempre disponibile poiché la sua professione non è semplicemente un mestiere ma una vita" (3). Ma sempre a partire dalla stretta di mano, dalla pacca sulla spalla, dal chiamare il malato per nome. Che non è un trito paternalismo, ma una forte espressione di rispetto della dignità umana e della malattia che la mina.

\section{Madre e figlio di fronte alla dialisi: il valore della comunicazione}

A tale proposito e a conferma e a testimonianza di quanto espresso, presento due storie illuminanti, seppure di senso opposto, ma comuni nella pratica del nefrologo. Una madre angosciata scrive: "Oggi ho avuto la triste notizia che mio figlio - 26 anni - sarà sottoposto a dialisi. È un dramma per lui, per me, per la famiglia. Non so come potremo affrontare questo evento, anche perché siamo impreparati, chiediamo aiuto. Offro la mia sofferenza perché egli possa reagire e sperare in un futuro migliore, magari con un trapianto renale" (4). Madre e figlio si presentano al nefrologo prima di iniziare la pratica dialitica. Ed ancora la madre scrive: "Il nefrologo nello stringere la mano a mio figlio e a me e nello spiegarci $i$ termini della tecnica terapeutica, ha infuso in noi, con il suo semplice ma apprezzato gesto, una grande fiducia e speranza che hanno sostituito l'iniziale disperazione. Con il nefrologo mio figlio ha fatto proprio il principio secondo cui il miglior atteggiamento verso un improvviso evento negativo è costruire un'immunità interiore tale da non essere intaccata dalle vicissitudini della vita. L'atteggiamento e le parole del nefrologo, anche con la prospettiva di essere subito inserito nella lista di attesa trapianto, hanno ridato a mio figlio la gioia di vivere, di amare, di valorizzare la propria esistenza anche se tribolata. Malgrado tutto, egli ha riacquistato la bellezza del sorridere alla vita nel suo distillato di quotidiana accettazione delle avversità fisiche, senza cadere in effervescenti emozioni e/o in decadenti catastrofismi". Dopo un anno è stato trapiantato.

Una giovane collega, il cui padre dializzato è stato ricoverato in Rianimazione in coma per un'estesa emorragia cerebrale, mi fa scivolare tra le mani una lettera di cui riporto, con il suo consenso, la parte finale. "... Avrei voluto sperare, ma non mi è stato possibile. Sentivo il bisogno incontrollabile di farlo ma tutto ciò mi è stato impedito dalle frasi fredde, spietate, insensibili di qualche collega. Che dolore ricordare quelle affermazioni: "puoi solo augurarti che tuo padre finisca il prima possibile... se mai si dovesse svegliare sarebbe peggio di un film dell'orrore... ma non ti preoccupare, non durerà ancora per molto». Ero scioccata, offesa per la crudezza con cui mi si parlava, sdegnata e terribilmente delusa per la terminologia usata proprio da colleghi sui quali avevo limpidamente riposto l'ultimo flebile alito di speranza. Mi sono chiesta più volte perché quella notte sia stata così verbalmente aggredita e il mio dolore invece che lenito veniva infinitamente amplificato.
Perché tanta violenza nelle frasi pronunciate? Tutto ciò stride con la figura del medico che con scienza e coscienza informa il paziente (e parenti) circa le condizioni cliniche senza mai usare terminologie traumatizzanti e senza mai escludere ambiti di speranza. Nel nostro lavoro non basta la perizia scientifica e professionale: occorre Amore" (5). Certo, amore. Una parola che ha sempre intriso il suo significato nel divenire storico del guarire.

\section{Il medico Eutichio: una brava persona}

Una recente scoperta archeologica in una piazza di Rimini ha messo in evidenza uno studio medico della prima metà del III secolo d.C. Lì, sulla parete di una saletta un paziente ha inciso la scritta: "Euthychius homo bonus hic habitat. Hic sunt miseri" (Qui abita Eutichio, una brava persona. Qui si ritrovano gli infelici). Certo, la collega figlia del nefropatico in rianimazione avrebbe voluto incontrare l'Eutichio della situazione, ma così non è stato, anche se per esperienza diffusa, di Eutichio ce ne sono molti tra la classe medica e nefrologica. La prima testimonianza ne è una dimostrazione poiché il nefrologo ha saputo ascoltare. Il medico sa 'ascoltare' il silenzio prolungato di un malato. È il silenzio di un depresso o il silenzio del malato con insufficienza cardiorespiratoria la cui conseguente dispnea gli rende il parlare estremamente penoso. È un silenzio che va rispettato in nome di un corretto rapporto medico-malato. Questo rapporto nella sua estensione è oggi affiancato dalla medicina narrativa, un approccio che si basa sulla narrazione della propria malattia e del proprio disagio. La medicina narrativa fondata da Rita Charon (6) considera $\mathrm{i}$ racconti della persona malata come punto nodale della relazione medico-malato per far emergere l'interpretazione di ciò che la medesima riferisce (7). Applicata ai nefropatici in dialisi fa emergere le proprie emozioni, le storie trascorse, le paure, le ansie, le aspettative, il disagio che la condizione di dializzato comporta e con il quale convive. $\dot{\mathrm{E}}$ insomma il contrario del non-ascolto.

\section{Il non-ascolto mina ogni comunicazione tra uomini}

Secondo l'epistemologo Massimo Baldini, il non-ascolto mina dalle fondamenta la comunicazione tra uomini (8). La difficoltà dell'ascolto, se traslata al rapporto medico-malato, crea un'asimmetria relazionale a scapito del più debole: il malato. Al contrario, l'attenzione del medico nell'ascolto del malato significa abbandonare temporaneamente i propri schemi, le proprie certezze, le proprie conoscenze e convinzioni per immergersi nelle categorie, nelle intenzioni che strutturano la comunicazione del paziente. Per rendere simmetrico il dialogo. Nelle parole di Cousins, citato da Baldini, c'è l'essenza di questa simmetria: "C'è un tipo di retta delineata dal medico quando si china sulla solitudine del malato, sulle sue paure, sul suo dolore, sul senso di mortalità che scaturisce dall'ignoto e quando la mano del medico sulla spalla e sul braccio del malato diventa una protezione contro le tenebre" (9). Ancora la mano del medico che stringe, che dà la pacca sulla spalla, che offre vicinanza, che manifesta condivisione. Ripeto, non 
è questione di paternalismo, ma di protezione dalle negatività della malattia. Così come l'ascolto. Per dirla ancora con Baldini, un ascolto del dializzato, con la piena fioritura dei sensi, un ascolto non opacizzato, non affievolito o intorpidito è il presupposto di ogni vero dialogo, di ogni comunicazione piena (10). Il tutto inserito in un contesto di bioetica. Poco si parla di questa branca del sapere medico e ancor meno del suo inserimento nel curriculum del nefrologo, il quale è il più esposto ai problemi di etica medica. É egli attrezzato, ad esempio, ad affrontare il problema del traffico di organi? Giunge alla mia osservazione Feisal, giovane pachistano che ha venduto per necessità un rene a un nefropatico occidentale. Dopo poco tempo sviluppa un cancro del rene superstite. Resta la dialisi che pratica per un certo periodo in Italia. Due problemi. Il commercio di organi sembra travolgere ogni regola di umanità e dignità che pervadono la medicina dai tempi di Ippocrate. Sembra che ogni traccia di etica sia bandita in questo mercato di organi. La posizione della Transplantation Society (Aprile 2008) è molto chiara, stabilendo di "Prendere misure per proteggere i gruppi più poveri e vulnerabili del turismo trapiantologico e del mercato di tessuti e organi, con particolare attenzione al più vasto problema del traffico internazionale". Posizione netta ma non vincolante. La vicenda di Feisal ne è una testimonianza. Ancor meno vincolante ed efficace è la fondazione Organ Watch, un osservatorio sulla violazione dei diritti umani nella raccolta e distribuzione degli organi da trapiantare (11). Il secondo problema è l'organizzazione del nefrologo italiano di fronte ad una situazione nuova, o per lo meno inusuale: inviare un dializzato nella sua nazione dagli incerti profili assistenziali. Personalmente ho affrontato come tanti nefrologi la condizione opposta, cioè di nefropatici italiani che hanno fatto lo stesso percorso ma in senso opposto, per ricevere un rene che nel tempo non ha più funzionato. Tutti in partenza per o da bazar di organi: c'è chi compra e chi vende (12). E il medico di fronte al commercio di organi, di fronte a persone che lucrano sulla miseria dei poveri e sulle sofferenze dei malati, quale risposta bioetica può fornire? $\mathrm{Si}$ deve ricorrere ancora al Giuramento di Ippocrate che impone prima di curare e poi di organizzare la prosecuzione della cura? In ogni caso eliminare sempre il conflitto di interesse (13). Eliminazione che salpa sempre dalla stretta di mano che suggella in positivo il rapporto medico-malato.

\section{Riassunto}

La stretta di mano è un gesto essenziale nell'approccio al binomio medico-malato. Sociologi, psicologi medici e bioeticisti ne sottolineano l'importanza nella gestione iniziale della malattia: piccolo gesto per il medico, grande per il malato. Apparentemente controcorrente in un mondo in cui tutto è fast, la stretta di mano viene considerata la chiave giusta per aprire la porta della bioetica in medicina coniugata alla comunicazione medico-malato. Come testimonianza viene riportata la storia clinica di tre pazienti con problemi nefrologici, che fanno emergere la valenza della bioetica nella loro risoluzione ed il significato umano della sua assenza. Viene sottolineato il valore della comunicazione, come il disvalore del silenzio e del non-ascolto nel rapporto medico-paziente. Il non-ascolto mina dalle fondamenta la comunicazione tra uomini sani e malati. Al contrario l'ascolto del dializzato è il presupposto di un vero dialogo, di ogni piena comunicazione. Sono presentate le categorie della medicina narrativa ed il loro utilizzo nella bioetica nefrologica.

Parole chiave: Stretta di mano, Bioetica in dialisi, Medicina narrativa

Dichiarazione di conflitto di interesse: L'Autore dichiara di non avere conflitto di interessi.

Contributi economici agli Autori: L'Autore dichiara di non avere ricevuto sponsorizzazioni economiche per la preparazione dell'articolo.

\section{Bibliografia}

1. Buckman R. How to break bad news. A guide for health care professionals. Johns Hopkins University. Baltimore, 1992.

2. Prati PL. Piccole grandi cose. La stretta di mano in medicina. Cuore e Salute. 2000; 9: 278-9.

3. Russo G. Bioetica medica. Messina: Editrice Elledici, 1999; 28.

4. Timio M. Dializzati sì, ma non uomini marginali. In: Timio M. Stringi la mia mano. Vivere con speranza i tempi della prova e del dolore. Assisi: Edizioni Porziuncola, 2011; 33-42.

5. Capezzali G. Storia di amore filiale e di ordinaria insensibilità. Perugia: Bollettino Ordine dei Medici, 2005; 6: 20.

6. Charon R. Narrative medicine. Honoring the stories of illness. New York: Oxford University Press, 2006.
7. Benedetti F. La medicina narrativa e le dinamiche nascoste della mente. Roma: Carocci, 2013; 17.

8. Baldini M. Educare all'ascolto. Brescia: Editrice La Scuola, 1988; 81 .

9. Ibidem.

10. Ibidem.

11. Timio M. Migranti. Volti e storie dalle periferie del mondo. Assisi: Cittadella Editrice, 2014; 52-3.

12. Gazzaniga V. La medicina antica. Roma: Carocci Editore, $2014 ; 33$.

13. Kiellstrand CM, Dosseton JB. Ethical problems in dialysis and transplantation. Dordecht, Boston, London: Kluwer Academic Publisher, 1992; 91. 